\title{
THE HARALD CRAMÉR VOLUME
}

\section{Carl Philipson}

Sweden

Harald Cramér was nominated Professor in Actuarial Mathematics and Mathematical Statistics at the University of Stockholm in the late twenties. I959 a book was "dedicated to Harald Cramér on his sixty-fifth birthday, in recognition of his pathbreaking work in probability and statistics", with the title Probability and Statistics, The Harald Cramér Volume (Almqvist \& Wiksell, Uppsala I959; John Wiley \& Sons, New York). The development during these three decades can be described in Cramér's own words in a recently published „Éloge de M. Paul Lévy." "Pendant ces années" (the late twenties) "la théorie moderne des probabilités a commencé ce développement puissant qui, depuis cette époque, a continué sans cesse, et qui a fait de la théorie des probabilités une des branches les plus fécondes de l'analyse moderne, ayant les rapports intimes avec tous les domaines des mathématiques pures, et en même temps des applications pratiques dont l'étendue et l'importance vont toujours en croissant."

A few historical notes will be given as a background for the review of The. Harald Cramér Volume, I903-1934 Filip Lundberg formed the collective theory of risk. This theory is a particular part of the general theory of stochastic processes which latter was drafted, in its main lines, by Kolmogoroff, I93I and I933. (A stochastic process is constituted by a variable depending on chance which is simultaneously a function of one or more variates. For readers not used to this terminology such a variable may be exemplified with the total risk costs for a certain period paid for a group of policies issued by a fire insurance company. For simplicity, it is assumed that the group involves only agricultural risks, similar to each other and situated in the open country so that no conflagration can occur. Further we assume that during the period of observation no change occurs in the composition of the group neither through underwriting, nor through cancellation, nor through alteration. The risk costs of such a group depend on chance and 
are, further, a function of time, the fire risk being, e.g., not the same during dry and wet years.) r925 Paul Lévy published Calcul des probabilités, where i.a. he studied Laplace-Liapounoff's theorem (according to which the total of a great number of random variables under certain conditions are approximately distributed with a normal distribution function) making use of so-called characteristic functions (these represent in the space of non-real quantities the image of distribution functions). Cramér published, in 1928, On the composition of elementary errors, where i.a. a better approximation to the distribution function of the sum of independent random variables was treated, and in 1930 On the mathematical theory of risk, based on Lundberg's theory and involving an extension of this theory. In $1932 \mathrm{~F}$. Esscher published a paper in the lastmentioned field with the title On the probability function in the collective theory of risk. Independently of this development the English school-'Student', Fisher, Pearson K. and E.G.-worked out methods for estimating parameters from numerical results and for testing hypotheses. By this time these methods had not in a rigorous way been theoretically founded. Harald Cramér published, in 1945, Mathematical Methods of Statistics (Uppsala, I945; Princeton Math. Series, I946). In this book the prevalent methods of statistical analysis were founded on a strict basis with the use of the developments in the theoretical field during the nearest previous decades. Also in other respects our knowledge of the theory of mathematical statistics and its applications to different practical problems was materially enlarged through this book which is and will for long time remain a fundamental standard work for the whole field. During the latest decades Cramér's pupils have continued the evolution depicted, not least in the collective theory of risk. A brilliant expounding of this theory and its relation to the general theory of stochastic processes was given by Cramér in 1955 (Skandia Jubilee Volume). Cramér has further in I940 and I942 contributed to the general theory (Ann. of Math., 3I; Ark. f. Mat., Astr. och Fys., 28 B). In the paper of 1942 the so-called spectral analysis has been worked out and developed. This method involves the study of a process by the covariance between the random function attached to the process for different values of the parameter or parameters expressed by aid of the so-called 
spectral distribution function for the process. Also in $195 \mathrm{I}$ a contribution to the theory of stochastic processes was published by Cramér (Berk. Symp. on Math. Stat. and Probab.). Even as emeritus Cramér has managed to accomplish two papers (I959) on the prediction problem for stochastic processes (Ark. f. Mat., 4:6; Publ. de l'Inst. de Stat. de l'Univ de Paris) with the application of the spectral analysis.

The Harald Cramér Volume is of outstanding interest, though a complete understanding of many of the papers is difficult for readers who have not specialized in the scope of the particular paper. The reason why the book is of such an utmost interest is not only that it is written by the most prominent scholars of the whole world in the fields, in which Cramér's activity has centered, but also because many of the authors give a review of earlier results, add new results, and draw up lines of direction for further research within the scope of the particular paper.

Two of the papers, one by Neyman and another by Fix, Hodges and Lehmann, all from the University of Berkeley, where Cramér has lectured in several periods, have dealt with certain particular cases of testing hypotheses, where certain hypotheses may a priori be excluded. These papers can be said to import a new step on the evolutional line from the English school over Cramér's standard work referred to here above. Wilks (Princeton Univ.) has given an account of another method for testing hypotheses, where no assumptions with respect to the distribution of the population have to be made (contrary to the methods referred to in the previous lines). Such tests have been called non-parametric. G. Elfving (Helsinki) has expounded a theory for planning experiments, an advanced extension of Fisher's methods for such planning. Robbins (Columbia Univ.) describes a method for estimating the mean of a normally distributed variable by aid of a sequence of samples drawn from the population according to certain rules. Anderson (Columbia Univ.) has studied a problem of estimating non-measurable properties by aid of certain measures involved in the application to psychological and sociological research. Feller (Princeton Univ.) who has lectured during some years in Stockholm, has studied certain sums of random variables using combinatorial methods. Two of Cramér's Swedish pupils-Segerdahl and Wold--have 
written on problems concerned in the application of stochastic processes to particular fields. Segerdahl has in a lapidary style given a complete review of the results hitherto reached in the collective theory of risk. Wold has extensively written about ends and mean in econometric model building. Models used previous to Wold's paper in econometrics can be subdivided into models based on dependent variables, as introduced in 1943 by Haavelmo, and models based on independent variables, called explicit causal chains and mainly used by Wold. In his paper Wold suggests for certain applications causal chains of a new type called implicit such chains. A short section of Wold's paper-Sample, population, frame--is of particular interest (frame is taken to mean the domaine of application for a prediction based on a sample drawn from the population).

The remaining papers are all bearing upon the general theory of stochastic processes. Bartlett (Manchester Univ.) has studied the influence of this theory on the statistical analysis. After an interesting review the paper deals with time-series analysis, statistical inference for stochastic processes, the wider outlook resulting from stochastic process theory, the statistical analysis of point processes (where the individuals are labelled by one or more continuous parameters, such as age or spatial position, or even time itself). Bartlett has exemplified the discussion in one case by bacterial colonies stemming from individual independent parents and in another case by a spatial distribution of parent plants giving rise to progeny plants assumed to be independent apart from the mutual dependence in the same family arising from the position of the parents. Doob (Univ. of Illinois), one of the pioneers in the field, has given a theorem for a so-called Markov chain (this is a particular case of Markov processes which have the property that each value of the random function attached to the process depends on a value of this function for a lower value of the parameter, but is independent of the generation of this latter value of the function). Cramér's successor, Grenander, who also stands as the editor of the book, has treated some non-linear problems in probability theory, where he, inter alia, uses spectral analysis. Kac (Cornell Univ.) and Kendall (Magdalen Coll., Oxford) have studied certain particular processes, the former processes of stationary increments, the 
latter Markov processes, with regard to certain properties. Paul Lévy (France), whose name has been mentioned in the review given above, has constructed certain processes of Markov type, previously defined by Feller and Mc Kean, using the theory for the Brownial movement as a starting-point. Masani has alone written a paper concerning Cramér's theorem for the decomposition of a matrix-valued, monotonously increasing function into three components of different properties and together with Wiener another paper dealing with non-linear prediction. Rosenblatt's (Indiana Univ.) paper is bearing upon statistical analysis of stochastic processes involving estimates of the spectral density function, bias, mean square errors and certain asymptotic properties of such estimates. Also Tukey (Bell Telephone Lab. Inc.) has dealt with the measurement of spectra and affords an extensive analysis of the errors in the measures obtained. His list of literature shows that his theory can be applied to many different fields, namely aerodynamics, aeronautics other than aerodynamics, astronomy, atmospheric turbulence, other meteorology, oceanography and human control behaviour.

In spite of many positive impressions obtained in the perusal of the volume one cannot fail to notice that the editor must have had certain difficulties in balancing the comprehension and extension of the different papers. In some parts a more complete treatment is wanting, in other parts a more concentrated manner of writing might have been preferred. In a book of this type which is a combination of texts from different fields and by different authors such a lack of balance might be unavoidable.

Oné side of Cramér's activity as a professor has not been completely illustrated in the volume. The reason hereof may be easily understood. Cramér has in his training and teaching displayed an unparalleled ability to make things intelligible and real to his pupils and inspire them with enthusiasm. In this connection the reviewer may be allowed to offer You, Harald Cramér, the best thanks from all Your pupils, especially from those who have tried to apply the knowledge gained from You to different problems in fields outside the universities, not least to insurance problems. We thank You for having introduced us into the beautiful space of known mathematical relations, which space in such a high degree 
has been extended through Your eminent exploration activity, and express our congratulations on the celebration of this activity with the monumentum aere perennius which bears the name: The Harald Cramér Volume.

Published in Swedish, Gjallarhornet, I960. 